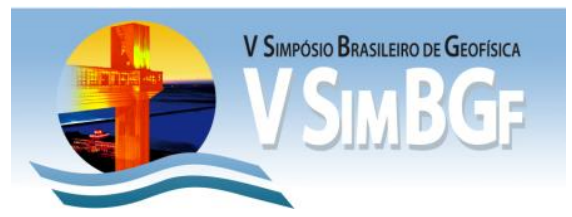

\title{
Determinação das oscilações livres da Terra com base no método de Rayleigh-Ritz
}

Luiz Gabriel S. de Oliveira, Natália V. de Oliveira e Carlos André M. da Silva - DCN/CEUNES/UFES

Emine Bayran - Cumhuriyet Üniversitesi - Turkey

Copyright 2012, SBGf - Sociedade Brasileira de Geofísica

Este texto foi preparado para a apresentação no V Simpósio Brasileiro de Geofísica Salvador, 27 a 29 de novembro de 2012. Seu conteúdo foi revisado pelo Comitê Técnico do V SimBGf, mas não necessariamente representa a opinião da SBGf ou de seus associados. E proibida a reprodução total ou parcial deste material para propósitos comerciais sem prévia autorização da SBGt.

\section{Resumo}

Este trabalho apresenta resultados preliminares oriundos da investigação teórica de oscilações livres esferoidais por meio da aplicação do método de Rayleigh-Ritz na determinação do campo de deslocamento associado. Um algoritmo computacional foi implementado com auxílio do pacote MATLAB. Os resultados mostraram-se satisfatórios e apresentaram a versatilidade desta técnica em estudos de sismologia de baixa frequência.

\section{Introdução}

Eventos sísmicos de grande magnitude costumam estar associados a propagação de ondas de corpo de baixa frequência, que são registradas em diversas estações sismológicas ao redor do mundo. Somado a este fato, ondas de superfície de longo período geradas pelos referidos eventos também trafegam ao longo da superfície da Terra, onde os efeitos da curvatura e rotação da influenciam nas características do registro (Dahlen 1968).

Em determinadas frequências discretas, tanto as ondas de corpo quanto as ondas de superfície induzem ao aparecimentos das oscilações livres ou modos normais de vibração (Aki \& Richards 2002), que podem ser entendidas numa primeira análise como a manifestação de padrões estacionários na propagação destas ondas. Basicamente, a Terra apresenta duas formas de oscilações livres: esferoidais e torodais, que dependem diretamente da ordem angular I e do harmônico $n$ do modo de vibração.

O fenômeno das oscilações livres constitui uma parte importante da Sismologia Global, pois permite refinar as informações sobre as propriedades elásticas e distribuição de densidades no interior terrestre, informações sobre o mecanismo de acoplamento mecânico e rotação diferenciada do núcleo interno em relação ao núcleo externo (Masters \& Gilbert 1981, Laske \& Masters 1999) e a possibilidade de cálculo de sismogramas sintéticos (Yang et. al 2010) pelo método da soma dos modos normais.

\section{Metodologia/ Problema Investigado}

A determinação das autofunções e autofrequências associadas às oscilações livres exige a resolução da equação de movimento no domínio da frequência (Dahlen \& Tromp 1998):

$$
\begin{aligned}
& -\omega^{2} \rho \vec{s}-\nabla \cdot \vec{T}+\left(4 \pi G \rho^{2} s_{r}\right) \hat{r}+\rho \nabla \phi \\
& \quad+\rho g\left[\nabla s_{r}-\left(\nabla \cdot \vec{s}+2 r^{-1} s_{r}\right) \hat{r}\right]=\overrightarrow{0}
\end{aligned}
$$

onde $\vec{s}$ é o campo de deslocamento, $s_{r}=\hat{r} \cdot \vec{s}, \omega$ a frequência angular $(\omega=2 \pi f, f=$ frequência), $\vec{T}$ o tensor de tensão de Cauchy, $\rho$ a densidade, $\phi$ o potencial gravitacional e $G$ a constante da gravitação universal.

Usualmente, a equação pode ser transformada num sistema de equações diferenciais ordinárias que podem ser resolvidas por um método numérico (Runge-Kutta, por exemplo) e que utiliza a representação do campo de deslocamento por meio de funções harmônicas esféricas.

Uma alternativa que apresenta-se computacionalmente mais atraente é a utilização do método de Rayleigh-Ritz, que possibilita o cálculo dos pares $\omega$ e $\vec{s}$ por meio de técnicas de álgebra linear. Isto é possível graças ao fato de que cada autofunção $\vec{s}$ pode ser representada como uma combinação linear de funções de base:

$$
\vec{s}=\sum_{\mathrm{k}} \mathrm{q}_{\mathrm{k}} \overrightarrow{\mathrm{s}}_{\mathrm{k}}
$$

que são resolvidas para os coeficientes de expansão $q_{k}$.

Assumindo um modelo de distribuição de densidades para a Terra com simetria esférica, sem rotação, perfeitamente elástico e isotrópico, definimos o funcional $\mathcal{J}=\mathcal{J}_{S}+\mathcal{J}_{T}$, onde $\mathcal{J}_{S}$ e $\mathcal{J}_{T}$ constituem as integrais esferoidais e toroidais:

$$
\begin{gathered}
\mathcal{J}_{S}=\int_{0}^{a} L_{S}(U, \dot{U}, V, \dot{V}) r^{2} d r \\
\mathcal{J}_{T}=\int_{0}^{a} L_{T}(W, \dot{W}) r^{2} d r
\end{gathered}
$$

definidas com base nas densidades Lagrangeanas esferoidal $L_{S}$ e toroidal $L_{T} . U, V$ e $W$ são as autofunções radiais associadas aos modos esferoidais $(U, V)$ e toroidais $(W)$.

Portanto, basta a definição dos variacionais $\delta \mathcal{J}_{S}$ e $\delta \mathcal{J}_{T}$ para que sejam determinadas os modos de vibração (esferoidais ou toroidais) associados a uma determinada frequência angular $\omega$.

No contexto apresentado acima, o presente trabalho pretende demonstrar a aplicação do método de RayleighRitz no estudo de alguns modos de vibração esferoidais 
da Terra. Maiores detalhes deste método e seu uso no estudo das oscilações livres da Terra podem ser encontrados em Dahlen e Tromp (1998).

\section{Resultados}

Para a realização deste trabalho, foram determinadas as oscilações livres esferoidais com base na distribuição de densidades predita pelo modelo PREM transversalmente isotrópico (Dziewonski \& Anderson 1981).

Como parâmetros de cálculo, o espectro de frequências adotado foi entre 0 e $30 \mathrm{mHz}$. A variação da ordem angular I foi entre 0 e 6000 . Já a ordem radial $n$ foi definida entre 0 e 100. As condições de contorno assumidas implicam na continuidade das trações e deslocamentos no interior da Terra, através de todas as suas interfaces, sendo nulas na superfície.

O método de Rayleigh-Ritz foi implementado com base no uso do pacote computacional MATLAB. O algoritmo construído segue a seguinte sequência de operações: i) leitura do modelo de densidades (PREM); ii) leitura dos parâmetros de cálculo das autofunções $U$ e $V$, além de suas respectivas derivadas radiais $\dot{U}$ e $\dot{V}(n, l$ e f); iii) determinação das integrais esferoidais; iv) determinação do variacional $\delta \jmath_{S}$; v) resolução das funções de base; vi) cálculo das autofunções radiais que compõem o campo de deslocamento $\vec{s}$ de cada modo esferoidal ${ }_{n} S_{\text {I com }}$ frequência específica $t$.

A Figura 1 representa diversos modos de oscilações esferoidais calculados com base no algoritmo desenvolvido com 0 uso do pacote computacional MATLAB.

Com base nos resultados, foi possível tecer as seguintes considerações:

1) $O$ método de Rayleigh-Ritz mostrou-se eficiente, do ponto de vista numérico, já que as soluções mostraram-se estáveis;

2) $O$ tempo computacional foi menor do que o verificado em simulações que envolveram códigos computacionais tradicionais (MINEOS e OBANI);

3) A versatilidade do método na investigação de estrutura interna da Terra, seja em todas as sua subdivisões $\left({ }_{0} S_{0} ; 9 S_{0}\right)$, seja em regiões específicas como 0 manto $\left({ }_{45} S_{2}\right)$, a interface núcleo-manto e a interafce núcleo externo-núcleo interno $\left({ }_{6} S_{2} ; 10 S_{2} ; 34 S_{2}\right)$.

\section{Discussão e Conclusões}

A implementação computacional, via MATLAB, do método de Rayleigh-Ritz mostrou-se eficiente na determinação de alguns modos de vibração esferoidais da Terra.

De uma maneira geral, o tempo computacional de cálculo foi menor do que o utilizado por outros códigos baseados em integrações numéricas, para o cenário aqui explorado. Portanto, foi possível calcular um maior número de campos de deslocamentos, em determinadas frequências discretas, num tempo relativamente mais curto.

Etapas futuras desta pesquisa pretendem: i) adaptar o método para a determinação das oscilações toroidais; ii) adicionar o efeito da rotação; iii) adicionar o efeito da elipsidade do planeta; iv) adicionar o efeito da anisotropia e v) calcular sismogramas sintéticos por meio do método da soma dos modos normais, baseado nos valores de deslocamentos e frequências correspondentes.

\section{Agradecimentos}

Ao Departamento de Ciências Naturais da Universidade Federal do Espírito Santo pela estrutura computacional utilizada.

\section{Referências}

Aki, K. \& Richards, P.G. 2002. Quantitative Seismology. $2^{\text {nd }}$ ed. University Science Books, California, USA.

Dahlen, F.A. 1968. The normal modes of a rotating, elliptical Earth. Geophys. J. Roy. Astron. Soc., 16: 329367.

Dahlen, F.A. \& Tromp, J. 1998. Theoretical Global Seismology. Princeton University Books. Princeton, USA.

Dziewonski, A.M. \& Anderson, D.L. 1981. Preliminary reference Eath model. Phys. Earth Planet. Int., 25: 29356.

Laske, G., \& Masters, G. 1999. Limits on the Rotation of the Inner Core from a new Analysis of Free Oscillations. Nature, 402: 66-68.

Masters, G., \& Gilbert, F. 1981. Structure of the inner core inferred from observations of its spheroidal shear modes. Geophys. Res. Lett., 8: 569-571.

Yang, H-Y., Zhao, L. \& Hung, S-H. 2010. Synthetic seismograms by normal-mode summation: a new derivation and numerical examples. Geophys. J. Int., 183: DOI: 10.1111/j.1365-246X.2010.04820.x 

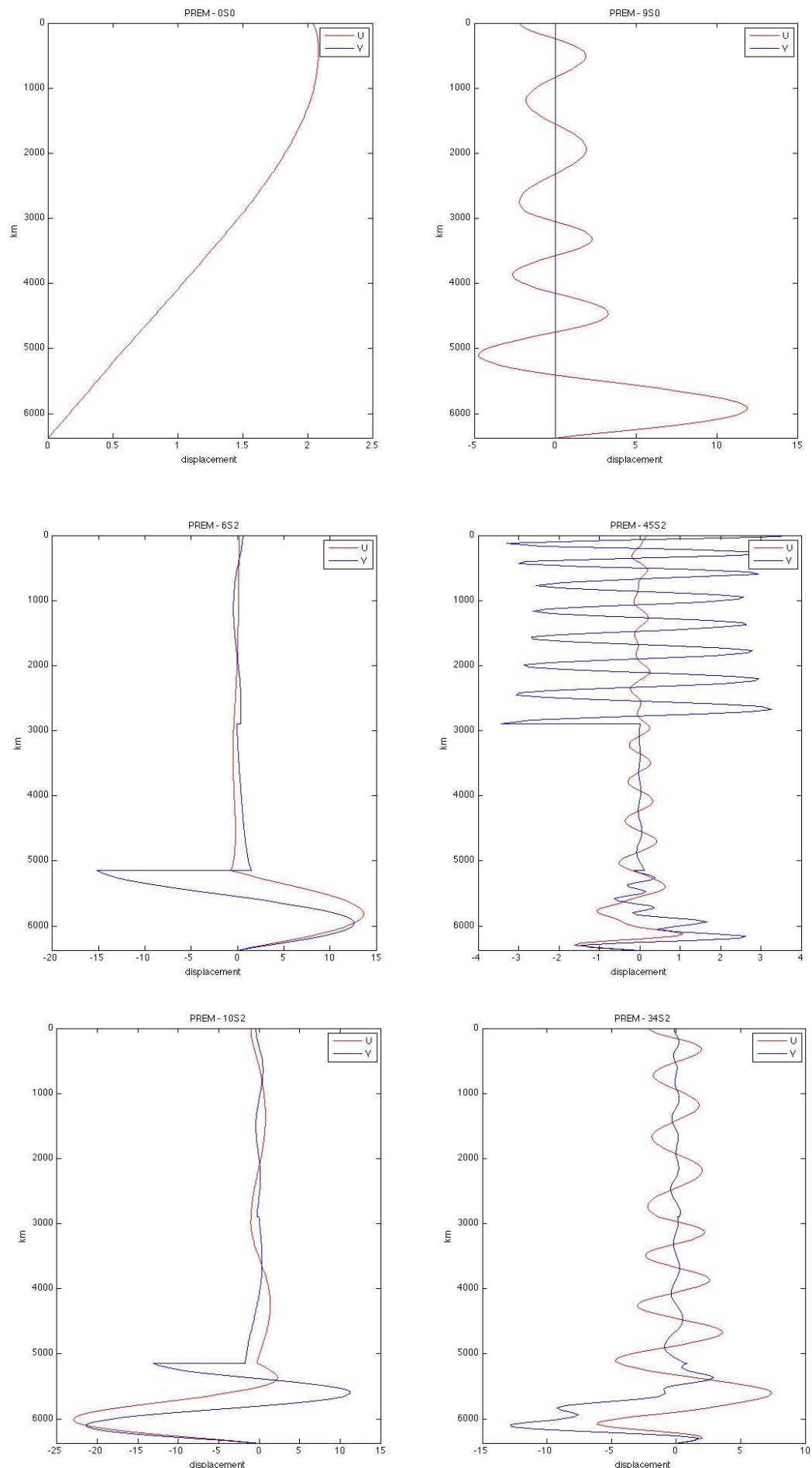

Figura 1 - autofunções radiais para alguns tipos de oscilações esferoidais da Terra, calculadas com base no método de Rayleigh-Ritz. Foi assumida a distribuição de densidades fornecida pelo modelo PREM. Valores de frequências teóricos: ${ }_{0} S_{0}(f=0.88 \mathrm{mHz}),{ }_{9} S_{0}(f=8.27 \mathrm{mHz}),{ }_{6} S_{2}(f=2.41 \mathrm{mHz}),{ }_{45} S_{2}(f=15.99 \mathrm{mHz})$, ${ }_{10} S_{2}(f=4.04 \mathrm{mHz})$ e ${ }_{34} S_{2}(f=12.35 \mathrm{mHz})$. 